\title{
Técnicas experimentales para caracterizar materiales fotoconductores
}

Experimental techniques to characterize photoconductive materials

Cristian Daniel Arambulo Almendariz. ${ }^{1}$ \& Luis Santiago Carrera Almendáriz. ${ }^{2}$

Recibido: 16-05-2021 / Revisado: 27-05-2021 /Aceptado: 20-06-2021/ Publicado: 05-07-2021

\begin{abstract}
DOI: https://doi.org/10.33262/concienciadigital.v4i3.1793

Introduction. Photoconductive materials are those that, when exposed to electromagnetic radiation, vary their electrical conductivity. Its applications are diverse and highly valuable in science and industry. Among the experimental techniques used to characterize these materials are scanning electron microscopy, optical microscopy, ray diffraction, the tunneling effect, among others. Objective. It presents a documentary review of the experimental techniques for the characterization of photoconductive materials. Methodology. The methodology was qualitative, related to the review of scientific papers, articles and texts, which allowed establishing an approximate state of the art in this field of study. Results. Among the relevant results, it was found that the characterization depends on the nature of the material and the source of electromagnetic radiation that is used to stimulate it, such as the intensity of the light, the frequency, the number of photons, among other properties. it is possible, with due caution, to use photoconductivity as a diagnostic tool in the study of new materials and electronic devices. Also, it was observed that the majority of samples analyzed and classified reveal a tendency to increase photoconduction, in addition to the use of numerical methods to

1 Escuela Superior Politécnica de Chimborazo Facultad de Ciencias, Riobamba, Ecuador, cristian.arambulo@espoch.edu.ec_ORCID: 0000-0003-1915-0976

2 Escuela Superior Politécnica de Chimborazo, Facultad de Ciencias, Riobamba, Ecuador, luissantiago.carrera@espoch.edu.ec ORCID: 0000-0002-3262-5895
\end{abstract}


carry out virtual experiments assisted by computer simulations, whose usefulness lies in the corroboration of the results obtained. by the empirical and analytical route.

Keywords: characterization of materials, experimental techniques, photoconduction, conductivity, numerical methods.

\section{Resumen}

Introducción. Los materiales fotoconductores, son aquellos que, al estar expuestos a la radiación electromagnética, varían su conductividad eléctrica. Sus aplicaciones son diversas y muy valiosas en las ciencias y la industria. Entre las técnicas experimentales que se usan para la caracterización de estos materiales, se encuentran la microscopía electrónica de barrido, la microscopía óptica, la difracción de rayos, el efecto tunelamiento, entre otras. Objetivo. Presentar una revisión de tipo documental, de las técnicas experimentales para la caracterización de materiales fotoconductores. Metodología. La metodología fue cualitativa, relacionada con la revisión de trabajos, artículos y textos científicos, que permitieron establecer un estado del arte aproximado, de este campo de estudio. Resultados. Dentro de los resultados más relevantes se encontró, que la caracterización depende de la naturaleza del material y de la fuente de radiación electromagnética que se usa para estimularlo, como, la intensidad de la luz, la frecuencia, el número de fotones, entre otras propiedades. Es posible, con la debida cautela, utilizar la fotoconductividad como herramienta de diagnóstico en el estudio de nuevos materiales y dispositivos electrónicos. También, se pudo observar que la mayoría de muestras analizadas y clasificadas revelan una tendencia al aumento de la fotoconducción, además, del uso de métodos numéricos para la realización de experimentos virtuales asistidos por simulaciones computacionales, cuya utilidad radica en la corroboración de los resultados obtenidos por la vía empírica y analítica.

Palabras claves: caracterización de materiales, técnicas experimentales, fotones, fotoconducción, conductividad, métodos numéricos.

\section{Introducción}

La caracterización de materiales es una actividad que usa métodos de la Física y de la Química Analítica para llevar a cabo el desarrollo de sus actividades. El conocimiento que se tiene de las clases de materiales en la actualidad es muy extenso, y su caracterización está sometida a diferentes fenómenos, entre ellos, la fotoconductividad, la cual, es la temática central de esta investigación.

En lo que respecta a la clasificación, existen tres grandes grupos: los metales, los polímeros, y los cerámicos. Sin embargo, una clasificación más actualizada, presenta una lista más extensa que contiene materiales compuestos, electrónicos, inteligentes, nanomateriales, con memoria de forma, piezoelectrónicos, microelectromecánicos, entre otros. 
Para los materiales mencionados, los experimentos muestran un incremento importante en los órdenes de magnitud de comportamientos fotosensibles y fotoconductores de los compuestos, cuando se les hacía incidir radiación electromagnética, del orden de magnitud del espectro UV y visible. Entre los parámetros detectados con éxito, se encuentran: tiempo de vida, longitud de difusión, el producto tiempo de vida-movilidad, niveles de impurezas y nivel de Fermi, el cual es un parámetro mecánico cuántico.

También, se han encontrado evidencias significativas del aumento de la fotoconductividad, de micro cavidades de silicio poroso nanoestructurado. Al respecto Urreaga, Marín, Acquaroli, Comedi, Schmidt \& Koropecki, (2008) señalan: "se utilizó silicio poroso nanoestructurado para construir una microcavidad óptica que permite aumentar la fotoconductancia debido al confinamiento del campo eléctrico dentro de la microcavidad. El dispositivo está formado por una capa de espesor óptico igual a media longitud de onda separado por dos arreglos periódicos de capas que alternan su porosidad" (p. 113)

En particular, en esta revisión documental, se persigue como objetivo general, conocer los referentes, métodos, resultados y conclusiones acerca de las investigaciones, con el fin de poder realizar una clasificación de las diferentes técnicas para la caracterización de materiales fotoconductores. Asimismo, la interrogante principal de esta investigación, está referida a lo siguiente: ¿Qué técnicas de experimentación son las más usadas en la caracterización de materiales fotoconductores?

\section{Metodología}

En el presente artículo, se realiza una investigación dentro del enfoque cualitativo, de tipo documental, acerca de las diferentes técnicas experimentales para caracterizar materiales fotoconductores. De la revisión realizada para llevar a cabo el proceso metodológico, se tomaron en cuenta, las de caracterización y revisión aproximada de las investigaciones de cuarto y quinto nivel (trabajos de maestría y doctorado), y los artículos en revistas indexadas, haciendo hincapié en las particularidades de cada una, así como sus potencialidades y debilidades experimentales y aplicativas. También se tomó en cuenta la correspondencia de los resultados experimentales con los modelos analíticos y numéricos, además, se presentan otras técnicas de caracterización de los materiales, que seguramente serán de interés para el lector.

Los materiales generalmente se caracterizan tomando en cuenta sus propiedades físicas y químicas. Los métodos de caracterización de dividen en dos grandes grupos, clásico e instrumental. El método clásico a su vez puede ser dividido en dos técnicas protocolares. La primera consiste en la separación de los componentes que se pretenden analizar (estos componentes se denominan analitos), por medio de la precipitación, extracción y destilación. La segunda, es la "formación", que consiste en la reacción del analito con un reactivo, con el fin de formar un producto determinado (Faraldos \& Goberna, 2011, p.19). 
Después de esta etapa, se procede a realizar un análisis cualitativo, donde se determinan tanto las propiedades físicas, como las químicas del analito, entre ellas, el color, el punto de fusión, el punto ebullición, el olor, la solubilidad, la actividad óptica, el índice de refracción, entre otras.

Los métodos instrumentales, se pueden dividir en dos grupos, el primero basado en técnicas cromatográficas de separación de alta eficacia para gases y líquidos. Este proceso puede reemplazar la separación de analitos. El segundo, consiste en el estudio de propiedades de la materia relacionadas con la emisión, absorción, dispersión, y difracción de radiación electromagnética (espectroscópicas). Sin embargo, otras propiedades, como la conductividad eléctrica y térmica, el potencial de electrodo, la proporción carga-masa, entre otras, pueden ser también incluidas (Faraldos \& Goberna, 2011, p.22).

Son muchas las técnicas que se han venido desarrollando y aplicando en este campo de estudios. El conocido diagrama de Propst, usado con frecuencia en Ciencias de los Materiales, representa esquemáticamente sus diferencias en función de las señales de perturbación, y la respuesta del material. Estas están asociadas a los distintos fenómenos que ocurren en el proceso experimental: fotones, electrones, iones, partículas neutras, calor y efectos de campo (eléctrico, magnético, y electromagnético).

En los métodos de análisis espectroscópicos de señales, como uno de los campos que mayor número de técnicas posee, por ejemplo, las señales pueden ser de siete tipos y asociadas con la interacción de partículas (Faraldos \& Goberna, 2011, p.53). La que interesa a esta investigación, es aquella que usa fotones como señal de estímulo.

En la caracterización de materiales semiconductores amorfos, a través de las técnicas basadas en la fotoconductividad, Kopprio (2019), presentó una investigación que mostró las distintas técnicas de caracterización en estado estacionario. El objetivo de la misma, era medir la conductividad del material, cuando era iluminado con luz de energía mayor a su banda prohibida (gap), tal que con los resultados obtenidos se pudiera hacer una clasificación de materiales semiconductores para la aplicación en la industria de celdas fotovoltaicas de alta eficiencia. Es importante mencionar, que está investigación presentó una innovación notoria, en cuanto a los formalismos matemáticos obtenidos, los cuales permitieron la correspondencia entre las mediciones de fotoconductividad bajo determinadas condiciones experimentales, con la movilidad de deriva de los fotoelectrones, la longitud de difusión ambipolar de los fotoelectrones, y el tiempo común de recombinación de pequeña señal $\tau$.

El control experimental se realizó tomando en cuenta el dopaje, la temperatura, la deposición química de vapor asistida por plasma, la capacitancia de los electrodos y la radiofrecuencia (luz incidente). A continuación, se describen las técnicas empleadas, la metodología de medición aplicada, los resultados, y la contrastación con el modelo teórico.

SSPC (Fotoconductividad de estado estacionario bajo una tasa de generación uniforme). Descripción. Se implementó la fotoconductividad de estado estacionario bajo 
una tasa de generación uniforme (por sus siglas en inglés, SSPC). Se iluminó la región de los contactos con luz en estado estacionario a través de un láser He-Ne enfocado sobre la muestra con la ayuda de un espejo. La modulación del haz con un Ch a baja frecuencia. Medidas. Se midió de la señal alterna el primer armónico usando un amplificador lockin para la determinación de la fotoconductividad. Modificando la temperatura y la tasa de generación se obtienen estados a distintas energías estimulando la fotoconducción. Resultados. Modificando la temperatura y la tasa de generación se obtienen estados a distintas energías estimulando la fotoconducción. Contrastación con el modelo teórico. Para una densidad de estados correspondientes a un material dopado tipo n, las expresiones propuestas son consistentes con los resultados.

MPC (Fotoconductividad de estado estacionario bajo una tasa de generación uniforme modulada en el tiempo). Descripción. Se aplicó la fotoconductividad de estado estacionario bajo una tasa de generación uniforme modulada en el tiempo (por sus siglas en inglés, MPC). Se ilumina la muestra con luz polarizada que pasa a través de un modulador electro-óptico. Cuando se aplica una diferencia de potencial se induce corriente alterna que se mide con un amplificador lock-in. Medidas. Para valores de temperatura entre los $40 \mathrm{~K}$ y los $440 \mathrm{~K}$ los de frecuencia alta aumentan su energía y conductividad. Resultados. Para valores de temperatura entre los $40 \mathrm{~K}$ y los $440 \mathrm{~K}$ los de frecuencia alta aumentan su energía y conductividad. Contrastación con el modelo teórico. Para temperaturas mayores a los $100 \mathrm{~K}$ los valores de fotoconductividad crecen y la técnica es consistente con los modelos analíticos, incluso con los numéricos simulados.

SSPG (Patrón de interferencia estacionario). Descripción. Se implementaron las técnicas del patrón de interferencia estacionario (por sus siglas en inglés, SSPG). Se ilumina a muestra con un haz laser con polarización lineal, el cual es separado por dos espejos. A continuación, se atenúa con un filtro de densidad neutra. Luego se hacen que interfieran. Medidas. Se midió la fotocorriente por medio del chopeo a una baja frecuencia del haz, con la ayuda de un amplificador lock-in. Resultados. En las regiones iluminadas se encuentran zonas oscuras muy extensas, las cuales no son afectadas por la difusión, apareciendo zonas en donde la resistencia total aumenta, disminuyendo la fotoconductividad. Contrastación con el modelo teórico. Los resultados y los modelos sólo son consistentes usando los mismos coeficientes de capturas, para todas las regiones.

MGT (Patrón de interferencia móvil). Descripción. Se utilizó el patrón de interferencia móvil (por sus siglas en inglés, MGT). Un haz de luz polarizado es separado por dos espejos semiplateados. La intensidad de uno de los haces es atenuada con un filtro de densidad neutra. Se usan espejos para hacer coincidir los haces con la muestra. Medidas. Se midió la corriente alterna, por medio del chopeo del haz débil, con la ayuda de un amplificador lock-in. Resultados. Se obtienen valores muy buenos de fotoconducción para frecuencias altas, además, aumentan con la temperatura y la intensidad de iluminación. Contrastación con el modelo teórico. Bajo condiciones de tasas de temperatura y generación uniformes, los experimentos son consistentes con los resultados. 
MPG (Patrón de interferencia modulado en el tiempo). Descripción. Se aplicaron las técnicas del patrón de interferencia modulado en el tiempo (por sus siglas en inglés, MPG). Consiste un patrón de interferencia oscilante en el tiempo sobre un fondo de iluminación de mayor intensidad. Se obtiene atenuando uno de los haces linealmente polarizado con un filtro de densidad neutra, resultando su intensidad menor. El haz débil pasa por un modulador óptico que produce una rotación del plano de polarización. Ambos se hacen coincidir en la región de la muestra. Medidas. Se midió la fotocorriente (corriente fundamental), producida por la diferencia de potencial aplicada entre los contactos, con la ayuda de un amplificador lock-in. Resultados. Para frecuencias altas, campo eléctrico externo muy pequeño y períodos grandes, se produce fotoconductividad en niveles medios con respecto al método MGT. Contrastación con el modelo teórico. Bajo las condiciones experimentales en las cuales se encontró fotoconductividad, los modelos son consistentes.

VPG (Patrón de interferencia vibrante). Descripción. Se aplicaron las técnicas relacionadas con el patrón de interferencia vibrante (por sus siglas en inglés, VPG). Un haz de luz polarizado es dividido en dos usando espejos semiplateados. Uno de los haces es atenuado, resultando su intensidad mucho menor a la del otro. Estos se hacen coincidir en la región de contacto. Medidas. Se midió la fotocorriente a frecuencia fundamental inducida en la muestra, con la ayuda de un amplificador lock-in, luego que la señal es preamlificada. Resultados. Para estados extendidos, en donde se asume el modelo de captura y emisión múltiple de los portadores hay fotoconducción. Contrastación con el modelo teórico. Bajo las condiciones establecidas para la experimentación, los modelos son consistentes, incluso, resultan más aproximados que los correspondientes a MGT y VPG.

OPG (Patrón de interferencia oscilante) Descripción. Se aplicaron las técnicas del patrón de interferencia oscilante (por sus siglas en inglés, OPG). Muy similar al VPG, pues ambos patrones son oscilantes en el tiempo. Medidas. Se midió la fotocorriente AC, la cual debe ser aproximadamente proporcional a la corriente inducida por MGT, usando la ayuda de un amplificador Lock-in. Resultados. Para frecuencias suficientemente bajas, como consecuencia de la iluminación MGT, se produce un cambio de sentido en el giro del patrón de interferencia, lo que estimula la fotoconducción. Contrastación con el modelo teórico. Los modelos son consistentes, considerando la suposición de que la diferencia de potencial aplicada induce un cambio de fase en la luz polarizada sólo en sus direcciones principales.

Es importante mencionar, que, en esta investigación, se llevaron a cabo simulaciones numéricas, que se correspondían con cada una de las técnicas aplicadas. Para llevar a cabo las simulaciones se tomaron en cuenta los siguientes aspectos: coeficiente de captura de huecos igual al de electrones, sólo dos estados monovalentes, los coeficientes son los mismos para distintas regiones DOS. Además, se usaron parámetros de materiales consistentes con los experimentos, para la misma muestra. Las técnicas de simulación y simuladores usados se pueden consultar en el trabajo original. Se encontró que, para lo parámetros y condiciones estipuladas, los modelos experimentales, teóricos y numéricos, 
tenían una buena correspondencia. También, se hicieron ensayos usando técnicas de la Ciencia de Datos, por medio de algoritmos de enseñanza y aprendizaje. También se obtuvieron buenos resultados.

En el campo de la Química Analítica, se hace caracterización de polímeros fotoconductores mediante métodos experimentales de cromatografía con detección múltiple, técnicas de fluorescencia y dinámica molecular. En esta investigación, se usaron estas técnicas para estudiar en los polímeros, el incremento de la conductividad eléctrica, cuando reciben radiación electromagnética. Señala Peña (2014): “entre los polímeros conductores se encuentran aquellos que exhiben un incremento de la conductividad eléctrica cuando reciben una radiación electromagnética, lo que se conoce como fotoconducción. Este fenómeno encuentra aplicación práctica en fotocopiadoras, cámaras de televisión, detectores infrarrojos, fotómetros, e indirectamente en el proceso fotográfico" (p.14)

En lo que atañe a los resultados obtenidos, con respecto al comportamiento fotofísico de las muestras, se obtuvieron: espectros de excitación y emisión de todos los compuestos en diferentes medios, los tiempos de vida de los estados excitados, espectros de anisotropía de fluorescencia en matriz sólida de PMMA y de desactivación (quenching) de fluorescencia en disolución diluida. Estas medidas suministraron información sobre procesos como la formación de excímeros, altamente usados en la industria, y la Desarrollo y optimización de técnicas basadas en la fotoconductividad para la caracterización de semiconductores con aplicaciones fotovoltaicas. Se pretendía en esta investigación, conocer la expansión del rango de amplitudes de vibración, detectables ópticamente, y nuevas, con amplitudes de desplazamiento más grandes que el tamaño típico de la inhomogeneidades de la distribución de luz, usándose la del tipo gaussiana, la cual tiene la particularidad de vibrar armónicamente en el plano de polarización del fotoconductor.

También se ensayaron soluciones de tipo numéricas a través de la aplicación del método de Runge-Kutta, con lo cual se pudieron contrastar los datos experimentales de las corrientes alternas en los fotoconductores analizados. Los resultados obtenidos muestran que las técnicas aplicadas son adecuadas para caracterizar fotoconductores en materiales cristalinos, además, de proporcionar información sobre las vibraciones de la franja en un rango de amplitudes, extenso y lineal. Las ecuaciones numéricas, consistentes con los resultados experimentales, muestran que la generación de la corriente alterna, tiene su origen en asimetrías del campo externo, mostrando la validez de los métodos numéricos como alternativa para la caracterización de materiales.

La técnica de Fotovoltaje Superficial (SPV), implica la detección de cambios en el potencial de contacto entre un semiconductor y un electrodo (sonda Kelvin) colocado cerca de su superficie, a diferentes condiciones de iluminación. Bajo estas condiciones experimentales, se forma una región de carga espacial en la masa cerca de la misma, equilibrada por la carga que reside en sus estados. La luz incidente genera carga, que se redistribuye debajo del campo, cuya distribución en estado estable es sensible a la 
longitud de difusión ambipolar del semiconductor $L$, que a su vez se refleja en el SPV. La medición de $L$ implica escalonar la longitud de onda de la luz incidente y ajustar su intensidad después de cada paso para mantener un SPV constante. Cada una corresponde con una profundidad de absorción específica $1 / \alpha$ en el semiconductor, y el valor de $L$ se obtiene a través de la intersección de un gráfico de intensidad de luz versus profundidad de absorción. (Kronik y Shapira, 1999, p.6-7).

Comúnmente SPV se usa particularmente en materiales a granel, aunque se afirma que surgen inexactitudes cuando se usa en películas delgadas, siendo versátil, y configurable para medir la densidad del estado de la superficie y las longitudes de separación de carga en materiales, como grupos moleculares y puntos cuánticos. También forma la base de una microscopía de potencial de superficie (microscopía de fuerza de sonda Kelvin) cuando se combina con un sistema AFM. La técnica en estabilizar la fuerza electrostática entre la punta y el semiconductor, la cual se ajusta a cero mediante la aplicación de un potencial de polarización, cuyo valor se representa en función de la posición para producir un mapa de superficie (mapeo conforme).

Otra técnica, es la Recombinación Dependiente del Giro, está influenciada tanto por las propiedades del material como por parámetros externos. Una variable externa adicional, junto con la temperatura y la intensidad de la luz, es proporcionada por la posibilidad de alterar la cinética de recombinación mediante la inversión de giro. Así, al iluminar una muestra fotoconductora y escanear en un sistema de resonancia de espín de electrones (ESR), la condición de resonancia puede detectarse como una disminución de la fotoconductividad.

La técnica TRMC, conocida como la Conductividad Microondas de Resolución Temporal, consiste en medir los cambios en la reflectividad de microondas de un fotoconductor cuando se crean portadores fotogenerados en exceso, por un breve destello de luz. La reflectividad es proporcional a la densidad del portador libre, lo que produce una desintegración transitoria en muchos órdenes de magnitud de tiempo similar al método TPC (Pillai et al., 2019. p.1-10).

Savenije et al. (2020), para la técnica TRM, reportan: "nuestras mediciones de TRMC en las bicapas revelaron que los electrones en estados defectuosos cercanos al VB pueden ser excitados por fotones con una energía tan baja como 1,30 eV. Su excitación conduce a electrones libres en el CB que se someten a inyección de carga en el C60 produciendo una separación de carga de larga duración. Por el contrario, la excitación de MAPbI3 / Spiro-OMeTAD a 1,3 eV muestra una señal que decae rápidamente, lo que implica que los agujeros están localizados y no pueden transferirse a través de la interfaz al SpiroOMeTAD” (p.1)

La fotoconductividad persistente (PPC), es el fenómeno en el que la respuesta del semiconductor puede persistir durante mucho tiempo después de que se apaga la iluminación, estando asociada con defectos cristalinos. Se ha observado en muchos semiconductores, incluidos el silicio (Si), azufre, óxidos y calcogenuros (Vega, 2019 p.16). 
Esta importante propiedad tiene implicaciones en una serie de aplicaciones optoelectrónicas, incluidas las células solares, los dispositivos regrabables y los fotodetectores. Entre estos materiales, el $\mathrm{CdS}$ ha sido ampliamente reconocido como un excelente fotoconductor. Se realizó la sintetización de películas de CdS utilizando trietanolamina (TEA) como agente complejante y tiourea como fuente de azufre. La fotosensibilidad informada, caracterizada por una relación de corriente de luz a oscuridad, alcanza 106 bajo iluminación AM2 para muestras recién preparadas y 107 después de que estas muestras se hayan almacenado durante varias horas a $50^{\circ} \mathrm{C}$ (Ko et al., 2017).

La razón de este aumento permanece indeterminada, pero se sugiere que se debe al oxígeno quimisorbido en la superficie y los límites de los granos que sirven como centros de recombinación adicionales, lo cual es consistente con los hallazgos de Lee, Mun y JU (2021). El mismo grupo también logró películas con aproximadamente 109 de fotosensibilidad bajo luz AM1 y un tiempo de descomposición de $13 \mathrm{~h} /$ década utilizando el mismo método de síntesis anterior, pero variando ligeramente la relación entre la fuente de cadmio y azufre. A pesar de la cantidad de trabajo sobre el efecto PPC en CdS, nunca se ha estudiado sistemáticamente para confirmar la fuente de PPC o el mecanismo subyacente real.

La Espectroscopia Infrarroja permite el estudio de las interacciones entre la materia y la radiación infrarroja, esta radiación corresponde a la zona- del espectro electromagnético que incluye las longitudes de onda entre los 0,1 y 1.000 picómetros. Y a su vez esta zona esta subdivida en infrarrojo cercano, infrarrojo medio e infrarrojo lejano (Faraldos \& Goberna, 2011, p.139).

Mediante la espectroscopia IR es posible identificar especies químicas de la frecuencia a la que los diferentes grupos funcionales presentan bandas de absorción en el espectro asociado. Además, la intensidad de banda puede ser utilizada para obtener la concentración de cada compuesto en la muestra. Por último, al comparar los espectros IR de distintas muestras, se puede conocer si ambas poseen la misma composición (Faraldos \& Goberna, 2011, p. 147).

La espectroscopía IR posee algunas ventajas importantes, como técnica analítica. Permite caracterizar muestras en cualquier estado de agregación, esto implica que se pueden realizar estudios en sustancias sólidas, liquidas o gaseosas. Esta es una técnica sencilla y rápida, en la mayoría de situaciones se puede preparar la muestra, registrar el espectro y emitir un resultado en unos minutos y además con una alta sensibilidad (Faraldos \& Goberna, 2011, p.160).

Cuando las moléculas son excitadas por la absorción de radiación UV-VIS, se presenta un fenómeno conocido como fluorescencia, este es un proceso en el cual las moléculas de la muestra emiten energía en forma de fotones. La técnica usada para llevar a cabo este estudio es la Espectroscopia Ultravioleta Visible. Generalmente, esta transición se produce cuando se excita el material de su estado electrónico fundamental. Una de las características más vistosas del fenómeno es su sensibilidad inherente al mismo, que con 
frecuencia posee una magnitud mayor que en la espectroscopía de absorción (Peña, 2011, p.38).

Sin embargo, estos métodos se aplican en menor cantidad que los de absorción, debido al número relativamente bajo de muestras que son capaces de fluorescer de forma apropiada. Por esta razón, en sus comienzos, la técnica únicamente era usada en el estudio de sistemas biológicos. En la actualidad se ha ido implementando en otros tipos de sistemas, entre ellos los polímeros sintéticos. Esta técnica es una poderosa herramienta, altamente sensible y no destructiva, muy importante para conocer el comportamiento físico y químico de las macromoléculas. Puede ser implementado en varios niveles, desde una simple medida de la emisión en estado estacionario hasta llegar a intrincados estudios de resolución temporal (Peña, 2011, p.35).

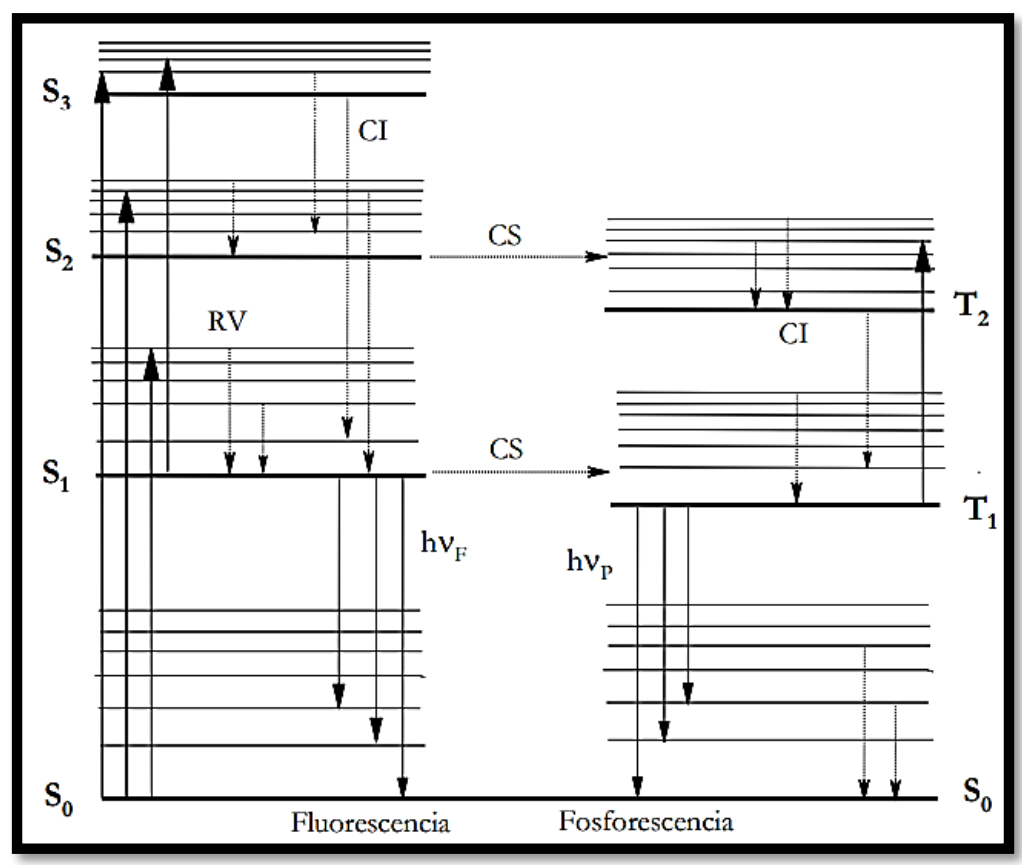

Figura 1: Esquemática de los diferentes procesos fotofísicos que pueden tener lugar tras la excitación inicial desde el estado.

Fuente: (Peña, 2011, figura 2.5, p.36)

De la investigación documental realizada se pudo encontrar que existen un conjunto de técnicas de caracterización de materiales fotoconductores, que presentan similitudes y diferencias en cuanto a sus procesos y aplicaciones.

En el caso de la caracterización de semiconductores:

1. La fotoconductividad de estado estacionario bajo una tasa de generación uniforme (SSPC), presenta que la estimulación de la fotocorriente en la muestra es favorable, pero presenta la limitación de que sólo es aplicable a materiales dopados n.

2. La fotoconductividad de estado estacionario bajo una tasa de generación uniforme modulada en el tiempo (MPC), para un rango de temperaturas estimado 
entre $40 \mathrm{~K}$ y $440 \mathrm{~K}$, existen valores de fotoconducción significativos, sin embargo, los modelos teóricos y experimentales son consistentes sólo para valores de temperatura $>100 \mathrm{~K}$.

3. Para la técnica basada en el patrón de interferencia estacionario (SSPG), se consigue fotoconducción muy baja, los modelos son consistentes sólo cuando los coeficientes de conductividad son iguales en todas las regiones.

4. Con la técnica del patrón de interferencia móvil (MGT), se consigue buena estimulación a la formación de fotocorrientes para altos valores de frecuencia, temperatura, e intensidad de luz. Los modelos con consistentes para tasas de temperatura y generación de portadores uniformes.

5. La técnica del patrón de interferencia modulado en el tiempo (MPG), muestra que, para frecuencias altas, campos eléctricos muy bajos, y periodos grandes, se genera buena fotoconducción en el material. Los modelos sólo son consistentes bajo estas condiciones.

6. El patrón de interferencia vibrante (VPG), para bajas frecuencias, aparece muy buena fotoconducción en el material, sin embargo, los modelos son consistente bajo suposiciones teóricas muy aproximadas, incluso, algunas veces erradas.

7. El uso de la técnica del patrón de interferencia oscilante (OPG), muestra que los modelos son consistentes, considerando la suposición de que la diferencia de potencial aplicada induce un cambio de fase en la luz polarizada sólo en sus direcciones principales

8. La técnica referida al uso de simulaciones computacionales, a través de métodos numéricos como: Monte Carlos, Runge-Kutta, y elementos finitos, proporciona buenos resultados que son consistentes tanto con el modelo teórico como con los resultados experimentales. Estos son más aproximados cuando se contrastan con los arrojados por MGT, OPG, y CMG.

En lo que respecta a los polímeros, además, de evidenciarse aumento en la fotoconductividad, por medio de la técnica expuesta, se ha logrado, la síntesis y purificación de los copolímeros usando AIBN. Este compuesto orgánico está formado por aminoisobutironitrilos unidos entre sí por un enlace $-N=N-$, por lo que se considera un azoderivado, que a temperatura ambiente se presenta como un sólido, en forma de cristales columnados de color blanco. También puede encontrarse en forma de polvo. Además, se logró su caracterización, usando cromatografía de tamaños con índice de refracción, y difusión de la luz a múltiples ángulos.

Sobre el comportamiento fotofísico de los copolímeros, se pudo estudiar la formación de excímeros intramoleculares y transferencia de energía, con el uso de la fluorescencia en estados estacionarios transitorios, reportándose espectros de emisión de todos los compuestos usados como muestra material, además, de los estados de vida excitados, y los espectros de anisotropía.

Estas medidas permitieron hacer una caracterización de los excímeros con respecto a su fotoconducción, además, de obtener información sobre procesos de formación, y 
eficiencia en la transferencia de energía intramolecular, que son magnitudes íntimamente relacionadas con la capacidad para conducir que presentan estos materiales.

En la caracterización de cristales, a través de la técnica de generación de fotocorriente provocada por patrones de iluminación dinámicos (photo-EMF), los resultados experimentales presentados por el autor, presentan cambios favorables en el fotoconductor hacia la conducción de portadores, cuando el material se encuentra en presencia de un campo eléctrico uniforme, externo y fuerte, lo cual se puede apreciar en el aumento paulatino de las vibraciones en un alto rango, sin necesidad de usar el mecanismos proporcionado por la técnica photo-EMF.

En cuanto al método numérico empleado (aproximaciones numéricas Runge-Kutta), se encontró que los modelos obtenidos permiten la descripción del transporte y captura de cargas en el fotoconductor, muy aproximadas a los resultados experimentales, y analíticos. Además, los resultados obtenidos por la vía de la simulación, muestran que la producción de fotocorriente alterna, posiblemente, se deba a la asimetría del campo interno.

Dentro de las posibles aplicaciones de las técnicas, lo más notable se menciona a continuación.

1. SPV ha encontrado gran aplicabilidad en la microscopía de potencia de superficie, y la producción de mapas de líneas equifotovoltaicas.

2. La recombinación dependiente de giro, con valores de $\mathrm{G}$ medios y cambios de conductividad, permite la obtención de información significativa y detallada para describir las vías de conducción asociadas con el spín, además, de aplicaciones a celdas solares y diodos LED.

3. TRMC, posee la virtud de que se puede llevar a cabo sin contacto con el material, lo que ha conllevado a aplicaciones para el estudio de muestras con grandes superficies, las cuales son fundamentales en la fotocatálisis. También se pueden encontrar aplicaciones en células solares, y sensibilidad de colorantes para industria química.

4. PPC, ha permitido que se puedan hacer caracterizaciones de materiales, aumentando la clasificación de los semiconductores, lo cual ha repercutido de manera relevante en las aplicaciones optoelectrónicas, los dispositivos regradables, y los fotodetectores.

5. IR, usa espectroscopia electrónica, para la identificación de especies químicas, concentración de los compuestos, y comparación de los espectros para hacer comparación de propiedades en las muestras.

6. La espectroscopia UV-VIS, tiene importantes aplicaciones en los fenómenos en donde la fluorescencia es una propiedad característica importante. Sin embargo, estos métodos se aplican menos que los de absorción, descansando este hecho en que pocos materiales son capaces de fluorescer. 


\section{Conclusiones.}

- La técnicas basadas en la aplicación de patrones de interferencia en estado estacionario y transitorio (SSPC, MPC, SSPG, MGT, MPG, VPG, y OPG), reportan consistencia con los resultados analíticos y simulados, permitiendo tanto la caracterización de materiales como aumentos en la fotoconducción de los mismos, sin embargo, los resultados más aproximados y más promisorios en cuanto al aumento de fotocorrientes en el material, resultaron corresponder con la técnica VPG, la cual se recomienda ampliamente para estados extendidos, en donde se asume el modelo de captura y emisión múltiple de portadores (bastante común en muchos materiales usados en la ingeniería e industria). Esta técnica, permite la creación y caracterización de nuevos materiales, entre ellos, los excímeros y copolímeros, de aplicaciones muy útiles y de bajo costo.

- Otras técnicas revisadas, SPV, ofrece ventajas en los materiales a granel, y desventajas en películas delgadas, pero es potencialmente aplicable en la separación de materiales y la caracterización de puntos cuánticos. Si se compara SPV, con las técnicas basadas en la aplicación de patrones de interferencia estacionarios y transitorios, y TRMC, las segundas presentan desventajas, pues necesitan obligatoriamente del uso de puntas de contacto material, lo cual no permite trabajar con polvos, agregados de materiales nanoporosos, y otras sustancias en las cuales es imposible definir electrodos.

- Todas las técnicas anteriores, son de difícil aplicación en el diseño y construcción de células solares, dispositivos regrabables y fotodectores, dados sus estados materiales, por lo que se recomiendan las técnicas PPC, dado que, resultan poseer altas propiedades optoelectrónicas, lo cual abarata costos de diseño y fabricación. Sin embargo, técnicas de menores costos y mayor versatilidad experimental, están relacionadas con la IR, las cuales resultan ser sencillas de preparar, además, de que se obtienen resultados más rápidos y sensibles para analizar. La desventaja que presentan, es que no son aplicables a materiales monoatómicos, ni a moléculas con la misma característica, pues los átomos que las conforman se hacen indistinguibles.

- Las técnicas de simulación computacional, dada su versatilidad y potencial, a través de los distintos softwares disponibles en la actualidad, y de la consistencia de los modelos simulados con los resultados analíticos y experimentales en el campo de la fotoconducción, pueden sustituir, con poca inversión monetaria, en todos los niveles, y en el ciclo de vida completo, el producto generado por la costosa experimentación.

- En la actualidad, no hay un entendimiento real de cómo funcionan esta mezcla de materiales, por la falta de modelos analíticos y experimentales aproximados. Esto podría ser solucionado a través de una simulación, o de la aplicación de algoritmos de aprendizaje, sin recurrir a una costosa prueba de laboratorio. La desventaja de estos métodos numéricos, está relacionada con el coste computacional, pues el proceso de modelado requiere de la elaboración de sofisticadas de mallas de elementos, las cuales, en ciertos casos, pueden ser de ordenes de magnitud muy 
grandes, provocando excesos de procesamiento de datos en el computador y posibles riesgos de daños irreversibles.

\section{Referencias bibliográficas.}

Faraldos, M., \& Goberna, C. (2011). Técnicas de análisis y caracterización de Materiales. Biblioteca de Ciencias del Consejo Superior de Investigaciones Científicas., 10(3), 513-520.

Ko, T. S., Chen, Z. W., Lin, D. Y., Suh, J., \& Chen, Z. S. (2017). Observation of persistent photoconductivity in Ni-doped MoS2. Japanese Journal of Applied Physics, 56(4). https://doi.org/10.7567/JJAP.56.04CP09.

Kronik, L., \& Shapira, Y. (1999). Surface photovoltage phenomena: theory, experiment, and applications. Tel Avid: Universtity Tel-Avid. Department of Physical Electronics.

Koprio, L. (2019). Desarrollo y optimización de técnicas basadas en la fotoconductividad para la caracterización de semiconductores con aplicaciones fotovoltaicas. Santiago: Universidad Nacional del Litoral. Grupo de Física de Semiconductores.

Lee, G., Mun, B., Today, H. J.-A. M., \& 2021, U. (2021). Observation of giant persistent photoconductivity on vanadium dioxide thin film device. Elsevier. https://www.sciencedirect.com/science/article/pii/S2352940720303425.

Peña N. (2011). Caracterización de polímeros fotoconductores mediante cromatografía con detección múltiple, técnicas de fluorescencia y mecánica y dinámica molecular. Alacalá: Universidad de Alcalá. Departamento de Química Física.

Pillai, S., Anderson, N., Wang, C., Bjuggren, J., \& Jevric, M. (2019). Validation of TimeResolved Microwave Conductivity (TRMC) as a screening tool for all-polymer solar cells.

https://bridges.monash.edu/articles/Validation_of_TimeResolved_Microwave_Con ductivity_TRMC_as_a_screening_tool_for_all-polymer_solar_cells/8313068.

Savenije, T., Thieme, J., \& Wei, Z. (2020). Using Time-Resolved Photoconductivity Measurements to Reveal the Urbach Tail and Two Photon Absorption in MHPs. Hal.Archives-Ouvertes.Fr. https://hal.archives-ouvertes.fr/hal-03030584/.

Urreaga, R., S., Marín, O., Acquaroli, L., Comedi, D., Schmidt, J., \& Koropecki, R. (2008). Aumento de la fotoconductividad y sintonización fina de la respuesta en microcavidades de silicio poroso nanoestructurado. Anales AFA, 20, 110-114.

Vega, A. (2019). Fuerza foto-electromotriz en películas de perovskita. Puebla: Instituto Nacional de Astrofísica, Óptica y Electrónica. Insituto de Óptica Aplicada.

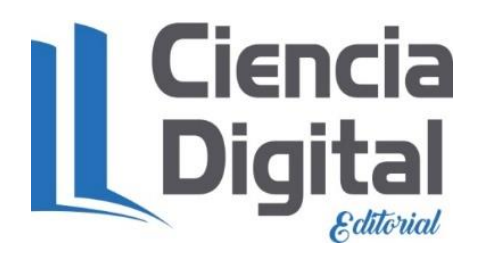




\section{PARA CITAR EL ARTÍCULO INDEXADO.}

Arambulo Almendariz, C. D., \& Carrera Almendáriz, L. S. (2021). Técnicas experimentales para caracterizar materiales fotoconductores. ConcienciaDigital, 4(3), 196-210. https://doi.org/10.33262/concienciadigital.v4i3.1793

\section{Ciencia}

El artículo que se publica es de exclusiva responsabilidad de los autores y no necesariamente reflejan el pensamiento de la Revista Conciencia Digital.

El artículo queda en propiedad de la revista y, por tanto, su publicación parcial y/o total en otro medio tiene que ser autorizado por el director de la Revista Conciencia Digital.

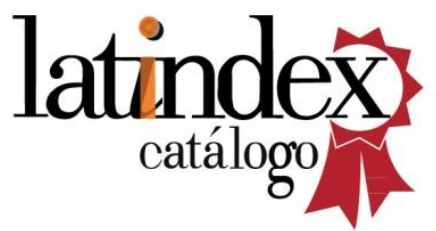

